\title{
Contributions to Bax insertion and oligomerization of lipids of the mitochondrial outer membrane
}

\author{
S Lucken-Ardjomande, S Montessuit and J-C Martinou*
}

Under many apoptotic conditions, Bax undergoes conformational rearrangements, leading to its insertion in the mitochondrial outer membrane as a transmembrane oligomer. At the same time, mitochondria undergo fragmentation and activated Bax was reported to localize to fission sites. We studied how lipid composition and membrane curvature regulate Bax activation. When isolated mitochondria were incubated with phospholipase $A_{2}$, which led to phosphatidylethanolamine and cardiolipin hydrolysis, tBid and Bax insertion were hindered. We thus studied in liposomes how phosphatidylethanolamine, cardiolipin, and its hydrolysis products affect Bax activation. Whereas phosphatidylethanolamine, a lipid with negative curvature, did not affect Bax insertion, it inhibited Bax oligomerization. Conversely, Bax insertion required cardiolipin, and was not blocked by cardiolipin hydrolysis products. These experiments support a direct role for cardiolipin in the recruitment and activation of Bax. To examine if the increase in membrane curvature that accompanies mitochondrial fission participates in Bax activation, we studied how liposome size affects the process, and observed that it was inhibited in small liposomes ( $\leqslant \mathbf{2 0 0} \mathbf{n m}$ diameter). Therefore, the localization of Bax to mitochondrial scission sites does not result from a preference for curved bilayers. Our experiments show that membrane properties can control the process of Bax activation, providing an additional level to the mechanisms of regulation of mitochondrial permeability.

Cell Death and Differentiation (2008) 15, 929-937; doi:10.1038/cdd.2008.9; published online 8 February 2008

The so-called mitochondrial pathway of apoptosis is central to the death of cells in response to many cytotoxic stresses. Much attention has, therefore, been drawn to elucidating the mechanisms that lead to permeabilization of the mitochondrial outer membrane (MOM). However, although it is known to be tightly regulated by pro- and antiapoptotic members of the $\mathrm{Bcl}-2$ family, it is for the moment unclear how these proteins coordinately control the release of mitochondrial apoptogenic factors into the cytosol. ${ }^{1,2}$ Among the proapoptotic members of the family, Bax and Bak appear to be the main effectors of MOM permeabilization. ${ }^{3}$

Under resting conditions, Bax exists as a soluble protein in the cytosol, perhaps associated with cytosolic retention factors, whereas Bak is a resident protein of the MOM, perhaps found as an inactive complex with VDAC2. ${ }^{3}$ After exposure to cytotoxic stresses, Bax and Bak are converted from inactive monomers to oligomeric transmembrane structures that promote MOM permeabilization. ${ }^{4-8}$ These conformational rearrangements occur in the MOM and certainly require a tight cooperation between Bax or Bak, their activators, and the lipid bilayer. ${ }^{9}$ Mitochondrial lipids could participate in these processes in various ways. First, they could be responsible for the specific recruitment of proteins of the Bcl-2 family to mitochondria. This appears to be the case for the activated $\mathrm{BH}$-only protein $\mathrm{tBid}$, which was proposed to specifically bind to the MOM via the mitochondria-specific phospholipid cardiolipin (CL) or one of its metabolites. ${ }^{10-12}$ Lipids could also assist in regulating the conformational rearrangements of $\mathrm{Bax}$ and Bak, and might contribute to their localization to specific regions of the MOM. Finally, lipids could directly participate in the mechanism of MOM permeabilization: as regulators of the association between cytochrome $c$ and the mitochondrial inner membrane (MIM), ${ }^{13}$ as actors in the remodeling of mitochondrial membranes that occurs during apoptosis, ${ }^{14,15}$ or as components of a mixed lipidic and proteinaceous pore. ${ }^{16-18}$ In this regard, lipids with negative intrinsic curvature, such as phosphatidylethanolamine (PE), were reported to inhibit the Bax-induced release of fluorescent compounds entrapped in liposomes. ${ }^{16,18}$ Interestingly, mitochondrial lipids appear to be modified early following apoptosis induction. In particular, CL was reported to be rapidly hydrolyzed ${ }^{10}$ and oxidized, ${ }^{13}$ which could provide signals for the onset of MOM permeabilization.

When cells undergo apoptosis, in addition to MOM permeabilization, mitochondria more or less coincidentally fragment. The role of mitochondrial fission for MOM permeabilization is not clear, but activated Bax displays a punctuate distribution at the surface of mitochondria and was reported to be localized at sites of mitochondrial scission. ${ }^{19}$ Bax could interact with proteins of the mitochondrial fission machinery and/or be recruited to these sites because the bilayer would display a particular feature promoting Bax oligomerization.

\footnotetext{
${ }^{1}$ Department of Cell Biology, University of Geneva, 30 quai Ernest-Ansermet, Geneva, Switzerland

${ }^{*}$ Corresponding author. J-C Martinou, Department of Cell Biology, University of Geneva, Sciences III, 30 quai Ernest Ansermet, 1211 Geneva 4, Switzerland.

Tel: + 412237964 43; Fax: + 412237964 42; E-mail: jean-claude.martinou@ cellbio.unige.ch

Keywords: apoptosis; mitochondria; Bax; lipids; cardiolipin; fission

Abbreviations: CL, cardiolipin; CLS, cardiolipin synthase; DCL, dilysocardiolipin; F/T, frozen and thawed; MCL, monolysocardiolipin; MIM, mitochondrial inner membrane; MOM, mitochondrial outer membrane; NAO, 10- $\mathrm{N}$-nonyl-acridine orange; PC, phosphatidylcholine; PE, phosphatidylethanolamine; PI, phosphatidylinositol; $\mathrm{PLA}_{2}$, phospholipase $\mathrm{A}_{2}$; PS, phosphatidylserine; shRNAs, small hairpin RNAs; TLC, thin-layer chromatography

Received 02.10.07; revised 19.12.07; accepted 08.1.08; Edited by M Piacentini; published online 08.2.08
} 
This could be a particular lipid composition or the curvature of the bilayer. Certain lipid domains that would facilitate membrane bending might form, similarly to what has been reported for phospholipase $D$ and mitochondrial fusion, ${ }^{20}$ or because of the separation of ordered and disordered domains. $^{21}$ Alternatively, highly bent membranes could facilitate Bax oligomerization.

We have undertaken a series of in vitro experiments to study the roles of the lipid bilayer in the processes of Bax insertion and oligomerization. We show that incubation of isolated mitochondria with phospholipase $A_{2}\left(P L A_{2}\right)$ completely prevents the insertion of tBid and Bax in the MOM. As only few mitochondrial lipids appeared to be significantly hydrolyzed by $\mathrm{PLA}_{2}$, we investigated whether any of these lipids had any impact on in vitro Bax insertion and oligomerization. We report that independent of $\mathrm{tBid}$, the insertion of Bax depends on the presence of CL and is not inhibited by the presence of $\mathrm{CL}$ hydrolysis products. Moreover, we show that $\mathrm{PE}$ specifically inhibits the process of Bax oligomerization. Finally, to investigate a putative role of mitochondrial fission in Bax activation, we analyzed the effect of the curvature of the bilayer using liposomes of various calibrated sizes, and report that Bax oligomerization is prevented in small liposomes (diameter $\leqslant 200 \mathrm{~nm}$ ).

\section{Results}

Incubation of isolated mitochondria with $\mathrm{PLA}_{2}$ prevents insertion of tBid and Bax in the MOM. Incubation of isolated mitochondria with recombinant caspase-8-cleaved Bid (tBid) triggers Bax activation and leads to MOM permeabilization. ${ }^{4}$ Activation of Bax involves exposure of an $\mathrm{N}$-terminal epitope, insertion in the lipid bilayer, and oligomerization. ${ }^{3}$ Recently, under these conditions, activation of Bax was shown to be abolished if mitochondria were predigested with proteinase $\mathrm{K}$ or trypsin, indicating that, in addition to tBid, mitochondrial proteins are necessary for Bax activation.22,23 These proteins could interact with Bax or could maintain or establish certain characteristics of the lipid bilayer required for the process. We therefore wanted to examine if mitochondrial lipids play a role in tBid-induced Bax activation. We incubated mitochondria isolated from HeLa cells with $\mathrm{PLA}_{2}$, an enzyme that cleaves fatty acyl chains of phospholipids at the position sn-2 of the glycerol moiety, and examined the efficiency of insertion of tBid and of endogenous Bax through classical alkali resistance tests (Figure 1a). ${ }^{5}$ Incubation with low amounts of $\mathrm{PLA}_{2}(0.3$ or $1.5 \mu \mathrm{g} / \mathrm{ml}$ ) decreased the degree of insertion of $\mathrm{tBid}$ and of Bax, while leaving the level of insertion of two other MOM proteins - Bcl- $\mathrm{x}_{\mathrm{L}}$ and Bak - unchanged. Results displayed in Supplementary Figure 1 show that the total amounts of tBid and of Bax associated with mitochondria were not diminished after the incubations, ruling out the possibility that our observations resulted from a degradation of the proteins or from their dissociation from the MOM.

As insertion of Bax is induced by tBid, the drop in Bax insertion could have resulted from the low amount of tBid inserted in mitochondria. Therefore, we attempted to restore tBid insertion in $\mathrm{PLA}_{2}$-treated mitochondria by increasing its concentration from 20 to $100 \mathrm{~nm}$ (Figure 1b). Under these conditions, whereas insertion of tBid was restored, Bax insertion remained lower than in the control. Although we cannot completely exclude the possibility that in $\mathrm{PLA}_{2}$-treated mitochondria, tBid could not adopt a particular conformation required for Bax activation, these results suggest that mitochondrial lipids directly influence the process of Bax activation. Thin-layer chromatography (TLC) analysis of mitochondrial lipids after incubation with $\mathrm{PLA}_{2}$ indicated that among the major mitochondrial phospholipids, $\mathrm{PE}$ and $\mathrm{CL}$ showed a significant decrease, while dilysocardiolipin (DCL) and fatty acids accumulated (Figure 1c). ${ }^{24,25}$ Moreover, during the time course of this incubation, mitochondria did not swell and did not show any gross change of morphology (data not shown).

Collectively, these experiments show that insertion of tBid and of Bax in mitochondria requires the presence of specific phospholipids or can be inhibited by the accumulation of their hydrolysis products. We therefore undertook a series of in vitro experiments to identify which mitochondrial lipid components modulate the efficiency of Bax activation.

CL is essential for Bax activation in vitro. $C L$ or its derivatives have been shown to be responsible for the specific recruitment of tBid to mitochondria, ${ }^{11,12}$ and previous reports suggested that $\mathrm{CL}$ is required for Bax to permeabilize synthetic liposomes. ${ }^{17,18}$ Therefore, we analyzed the importance of $\mathrm{CL}$ for Bax activation in vitro. We prepared liposomes containing phosphatidylcholine (PC), the most abundant cellular phospholipid, and decreasing concentrations of $\mathrm{CL}$, from 40 to $0 \%$. After ultracentrifugation, we examined the binding of $\mathrm{tBid}$ and of Bax to liposomes, as well as Bax oligomerization efficiency. For this purpose, we took advantage of a recently described in vitro assay based on the observation that partial resistance of Bax to trypsin digestion (i.e., detection of a trypsin-resistant Bax fragment, Tr-Bax) indicates the presence of active Bax oligomers. ${ }^{26}$ Results displayed in Figure $2 a$ show that the efficiency of binding of tBid and of Bax to liposomes and the amount of Tr-Bax formed diminished in parallel with the amount of CL. Even at $40 \% \mathrm{CL}$, tBid was still required for efficient binding and oligomerization of Bax (Supplementary Figure 2). Therefore, since the binding of tBid was also dependent on $\mathrm{CL}$, we prepared liposomes containing $\mathrm{PC}$ and the negatively charged phospholipid phosphatidylserine (PS), which can be bound by tBid, albeit with a lower affinity. ${ }^{12}$ We examined the efficiency of Bax binding and oligomerization in presence of 10,100 , or $500 \mathrm{~nm}$ tBid. Even at $500 \mathrm{~nm}$, and although tBid bound efficiently to liposomes, Bax binding was only moderately increased, and no Tr-Bax could be detected (Figure 2b). Similar results were obtained with liposomes containing PC and another negatively charged phospholipid phosphatidylinositol (PI, data not shown). Therefore, although we cannot exclude the possibility that in the absence of $\mathrm{CL}$, tBid cannot adopt a conformation required for its activity, CL appears to be important for the recruitment of Bax and its oligomerization in liposomes.

To circumvent the requirement of tBid and to unequivocally assess how lipids directly affect Bax activation, we examined the insertion of recombinant monomeric Bax in mitochondria 
a
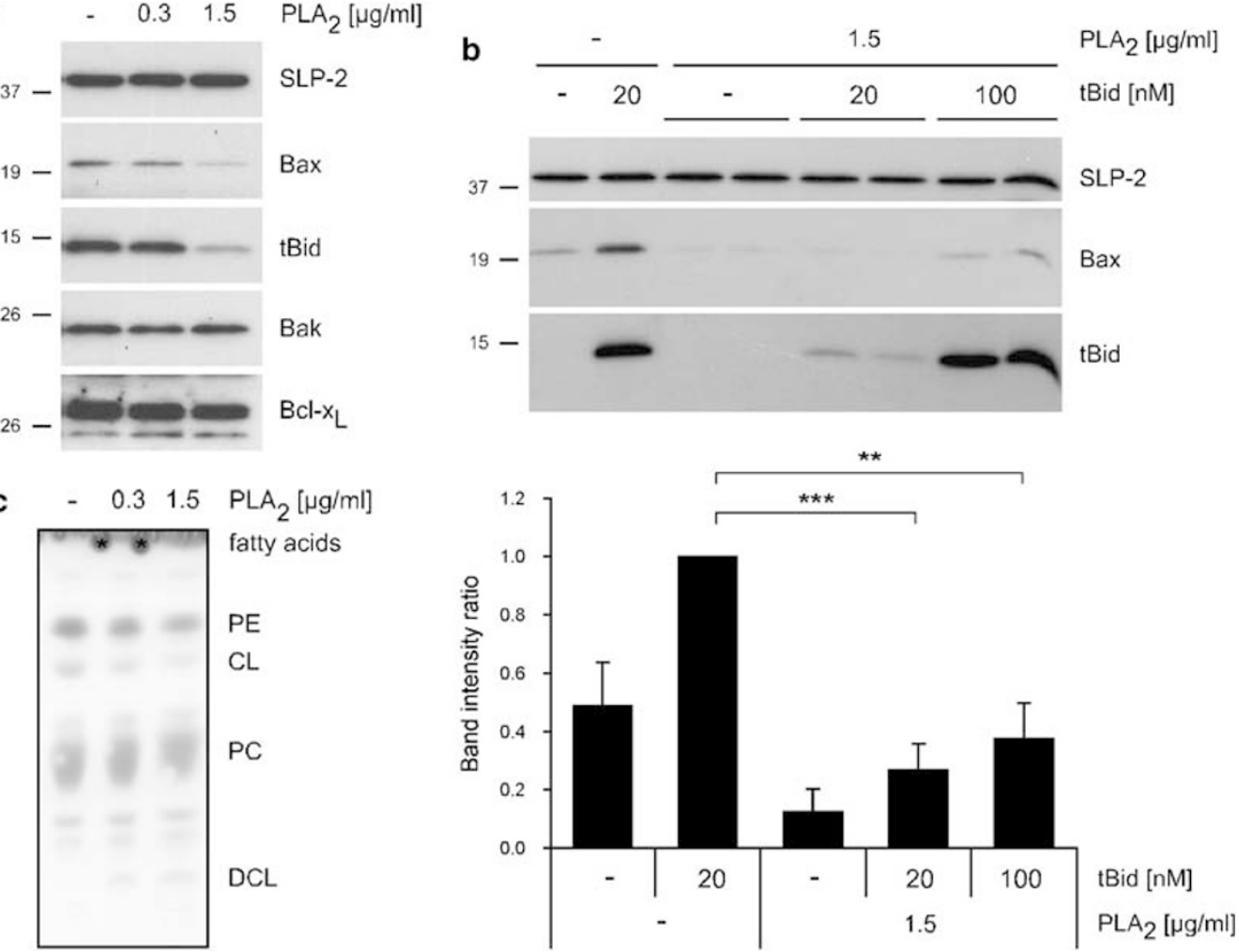

Figure 1 tBid and Bax do not insert in PLA -treated isolated mitochondria. Mitochondria were isolated from HeLa cells and incubated without or with PLA $(0.3$ or 1.5 $\mu \mathrm{g} / \mathrm{ml})$. Reactions were stopped by the addition of EGTA. (a) A further incubation in presence of tBid $(20 \mathrm{nM})$ was performed, and proteins inserted in the mitochondrial membranes were analyzed by immunoblotting after extracting peripheral proteins in an alkaline solution of sodium carbonate. An antibody directed against the MIM protein SLP-2 was used as a loading control. Blot is representative of three independent experiments. (b) Similarly, insertion of tBid and Bax in PLA $A_{2}$-treated mitochondria was assessed after incubation with 20 or $100 \mathrm{~nm} \mathrm{tBid.} \mathrm{PLA}$-treated samples were assayed in duplicate. For each condition, the intensity of the band corresponding to Bax was quantified by densitometric analysis and ratios to the intensity of the band obtained in the presence of tBid without any PLA $A_{2}$ treatment were calculated. Values are means of four independent experiments \pm S.E.M. ${ }^{* *} P<0.002,{ }^{* * *} P<0.0005$. (c) The lipid composition of $P_{2} A_{2}$-treated HeLa mitochondria was analyzed by TLC. Close to the fatty acid band are spots caused by the solvent front $\left(^{*}\right)$. The TLC is representative of seven independent experiments

isolated from yeast cells. We compared its efficiency of insertion in mitochondria purified from a wild-type and a mutant strain deleted for the gene coding for $\mathrm{CL}$ synthase (CLS, $\Delta c r d 1$ ), and therefore deficient in CL (Figure $3 a$ and b). Unlike what was previously reported, ${ }^{27}$ we observed a significant decrease in the amount of Bax inserted in CLdeficient mitochondria. However, in the initial study of Iverson and co-workers, ${ }^{27}$ recombinant Bax treated with the detergent octyl-glucoside, well known to promote artificial Bax oligomerization, ${ }^{28}$ was used, thereby probably preventing any examination of the dependence of Bax activation on lipids. Although the presence of tBid did not potentiate Bax activation, we also observed a decrease in the amount of tBid inserted in CLdeficient mitochondria (Figure 3a), in agreement with the fact that it was shown to be CL-dependent. ${ }^{12}$ These observations strongly support a direct requirement of $C L$ for the initial steps of Bax activation.

PE specifically inhibits Bax oligomerization in vitro. We then examined whether PE had any effect on tBid-induced Bax insertion in isolated liposomes. Results displayed in Figure 4a show that Bax inserted equally well in the absence or presence of PE $(20 \%)$, suggesting that PE digestion was not responsible for the lack of insertion of Bax in
$\mathrm{PLA}_{2}$-treated mitochondria. Recently, however, PE was reported to inhibit Bax-induced permeabilization of synthetic liposomes. ${ }^{18}$ We thus wanted to examine whether PE had any effect on the efficiency of Bax oligomerization in vitro. Using the previously described trypsin resistance assay, we examined the effect of PE on tBid-induced Bax oligomerization, gradually replacing $\mathrm{PC}$ with $\mathrm{PE}$ (Figure 4b). Up to $15 \%$, PE did not modify the amount of Tr-Bax formed, but when the concentration of PE was raised to $20 \%$, Bax oligomerization was inhibited, although the amount of Bax and tBid bound to the bilayer did not change. In comparison to the dependence on tBid, the specificity of the effect of PE on Bax oligomerization was striking (Supplementary Figure 2). As a first control, we used recombinant Fis, a mitochondrial protein with a single transmembrane domain. The insertion of Fis in liposomes was not abolished by the presence of PE, and Fis was equally sensitive to trypsin digestion using the two different liposomes (Figure 4c), indicating that PE did not simply impact on the activity of trypsin. To verify that the inhibition of Tr-Bax formation at 20\% PE was not linked to the reduction of PC (from 60 to $40 \%$ ), we analyzed, as a second control, the activation of Bax in liposomes containing $20 \% \mathrm{PI}$, and observed neither a difference in the efficiency of Bax binding 
a
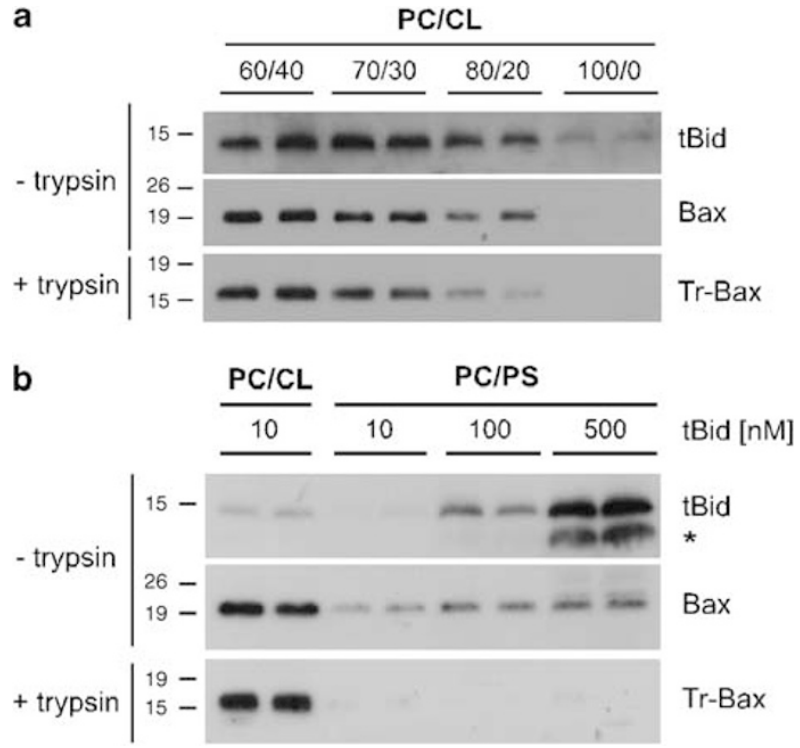

Figure $2 \mathrm{CL}$ is essential for Bax binding and oligomerization in vitro. (a) Liposomes containing PC and decreasing amounts of $\mathrm{CL}$ (from 40 to $0 \%$ ) were used in tBid-induced Bax binding (- trypsin) and oligomerization ( + trypsin) assays. (b) Similarly, PC/CL 60/40 and PC/PS 60/40 liposomes were assayed for Bax activation using 10,100 , or $500 \mathrm{~nm}$ tBid. The lower tBid band $\left({ }^{*}\right)$ is a minor Bid fragment obtained after caspase-8 cleavage of the recombinant protein. (a, b) Blots show duplicates and are representative of three independent experiments

a
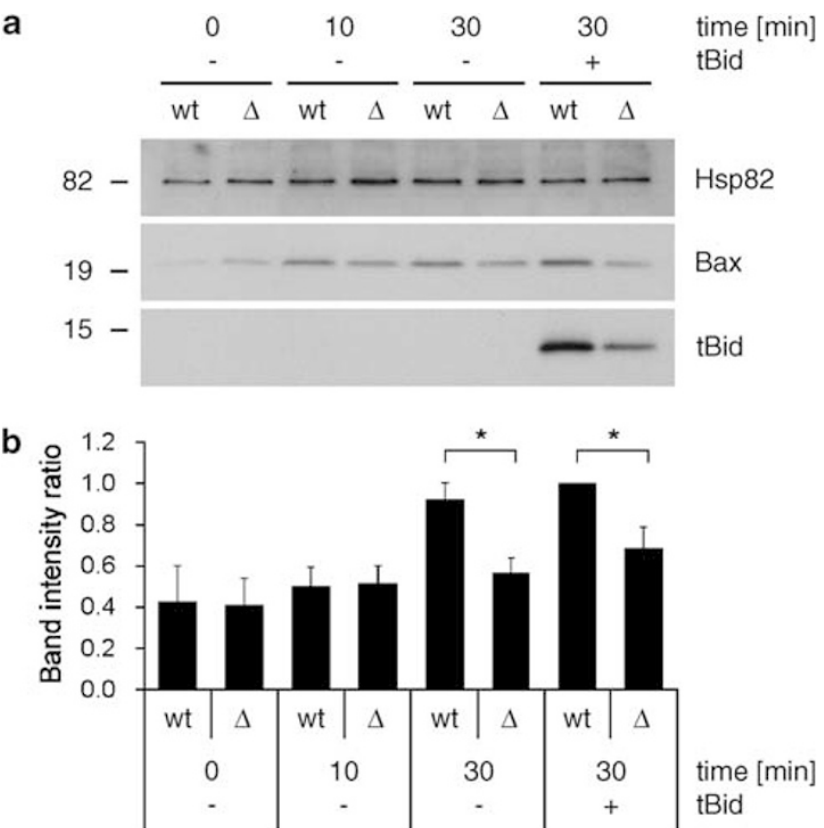

Figure $3 \mathrm{CL}$ is essential for Bax insertion in yeast mitochondria. Mitochondria were isolated from wild-type (wt) or CL-deficient $(\Delta)$ yeast cells, and incubated for various time points $(0,10$, or $30 \mathrm{~min})$ with recombinant $B a x(100 \mathrm{nM})$ in the absence $(-)$ or presence $(+)$ of tBid $(20 \mathrm{~nm})$. (a) The amount of Bax and tBid inserted in the membranes was assessed by immunoblotting the pellets obtained after a sodium carbonate treatment of mitochondria. An antibody directed against yeast $\mathrm{Hsp} 82$ was used as a loading control. (b) For each condition, the intensity of the band corresponding to Bax was quantified by densitometric analysis and ratios to the intensity of the band obtained in wild-type mitochondria after a 30 min incubation with tBid were calculated. Values are means of three independent experiments \pm S.E.M. ${ }^{*} P<0.03$ nor in the degree of tBid-induced Bax oligomerization (Figure 4d).

These experiments suggest that a decrease in the amount of $P E$ in the MOM cannot be responsible for the inhibition of tBid-induced Bax activation in isolated $\mathrm{PLA}_{2}$-treated mitochondria. Moreover, they highlight an effect of PE, a phospholipid with negative intrinsic curvature, on the efficiency of Bax oligomerization.

\section{CL hydrolysis products do not prevent Bax insertion in} vitro. To examine if products of $\mathrm{PLA}_{2}$ hydrolysis might be responsible for an inhibition of $\mathrm{Bax}$ and tBid insertion in isolated mitochondria, we first added linoleic acid (C18:2), the principal fatty acyl chain of $\mathrm{CL}$, to PC/CL liposomes and examined Bax activation in vitro. Linoleic acid potentiated the binding of tBid and Bax to liposomes, but did not impact on the efficiency of Bax oligomerization (Figure 5a), suggesting that the accumulation of fatty acids is not expected to prevent the insertion of Bax and tBid in the MOM.

$\mathrm{PLA}_{2}$ hydrolysis of isolated HeLa mitochondria also led to the formation of lysocardiolipin derivatives. Therefore, we examined if $\mathrm{CL}$ hydrolysis products would prevent Bax insertion in vitro. We hydrolyzed pure CL to obtain a mixture of $\mathrm{CL}$, monolysocardiolipin (MCL), DCL, and fatty acids, as shown by TLC analysis of the phospholipids (Figure $5 \mathrm{~b}$ ) and compared the efficiency of Bax activation using untreated or PLA $\mathrm{A}_{2}$-treated CL (Figure $5 \mathrm{c}$ ). The binding and insertion of tBid and Bax were similar in the absence or presence of lysocardiolipin derivatives, suggesting that formation of $\mathrm{CL}$ hydrolysis products was not responsible for the inhibition of Bax insertion in $\mathrm{PLA}_{2}$-treated mitochondria. Moreover, further digestion of CL to DCL and fatty acids did not allow any Bax-binding to the liposomes (data not shown). Therefore, complete digestion of $\mathrm{CL}$ to $\mathrm{DCL}$ in the MOM of isolated mitochondria (which most often appears to be the case upon $\mathrm{PLA}_{2}$ digestion in vitro) is probably responsible for the lack of Bax insertion following $\mathrm{PLA}_{2}$ treatment. Results displayed in Figure $5 \mathrm{c}$ also indicated that CL hydrolysis led to an inhibition of Bax oligomerization. This could be linked to a modification of some characteristics of the lipid bilayer, or to a decrease in the $\mathrm{CL}$ level, below a certain threshold required for Bax oligomerization. Therefore, whereas MCL, perhaps in combination with fatty acids, can compensate for the partial loss of $\mathrm{CL}$ and restitute efficient Bax insertion in vitro, it cannot sustain Bax oligomerization.

Bax does not oligomerize in small isolated liposomes in vitro. In addition to lipid composition, the shape of mitochondrial membranes might have an impact on Bax activation. Recruitment of Bax to mitochondrial fission sites ${ }^{19}$ could result (i) from its interaction with mitochondrial fission proteins, (ii) from its binding to specific lipid microdomains, or (iii) from a preference of Bax for curved bilayers; mitochondria typically have a diameter of $500-700 \mathrm{~nm}$, and mitochondrial constriction sites in yeast were reported to be $\sim 100 \mathrm{~nm}$ in diameter. ${ }^{29}$ To test the latter possibility, we analyzed the efficiency of Bax oligomerization in vitro using liposomes of different sizes. We prepared liposomes with a composition reflecting that of the MOMs (PC/PE/PI/CL/ cholesterol 48/25/12/6/9), ${ }^{30}$ and either performed a series 
a

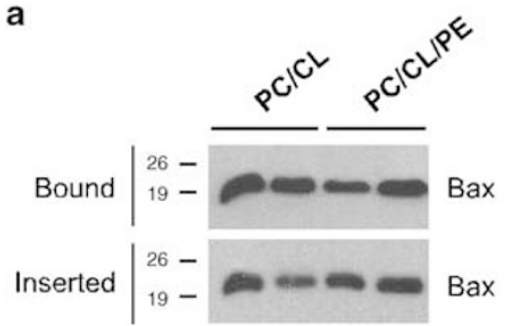

b

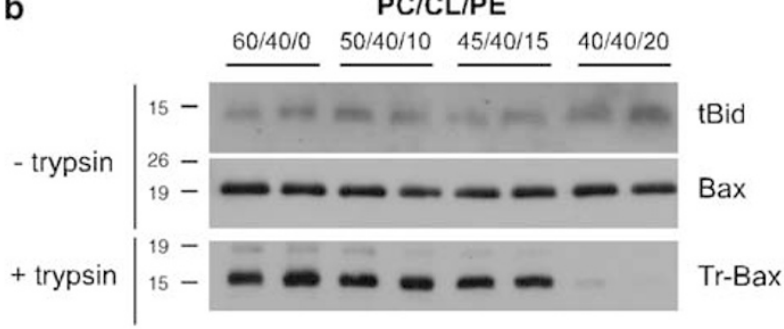

C

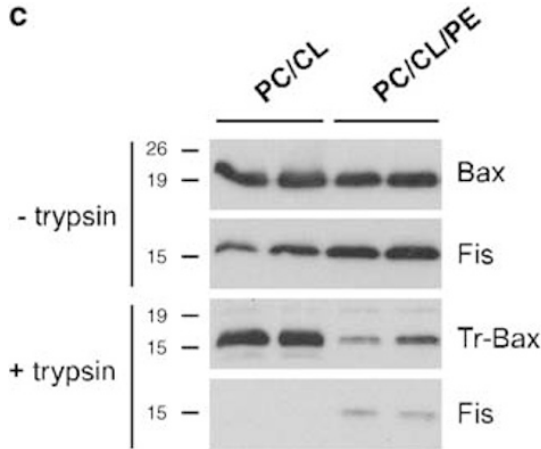

d

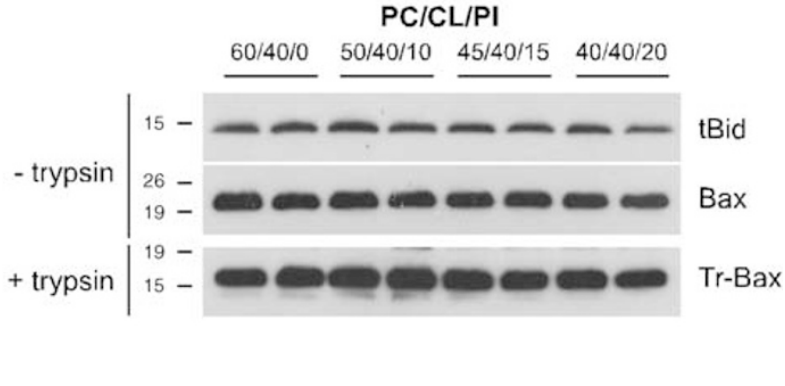

Figure 4 PE specifically inhibits Bax oligomerization in vitro. (a) PC/CL 60/40 or PC/CL/PE 40/40/20 liposomes were incubated with Bax and tBid. The insertion of Bax in the bilayer was assessed after extracting peripheral proteins in a solution of sodium carbonate and analyzing the pellets obtained by immunoblotting. (b, d) Liposomes containing PC, CL, and increasing amounts of PE or PI were used to analyze the efficiency of tBid-induced Bax oligomerization in vitro. The amount of Bax and tBid bound to the liposomes was assayed by immunoblotting (- trypsin), as well as the fraction of Bax oligomerized ( + trypsin). (c) As a control, the binding of recombinant Fis (50 nM) to $\mathrm{PC} / \mathrm{CL}$ 60/40 and PC/CL/PE 40/40/20 liposomes was tested in vitro, as well as its resistance to trypsin degradation. (a-d) Results are representative of at least three independent experiments. All blots show duplicates

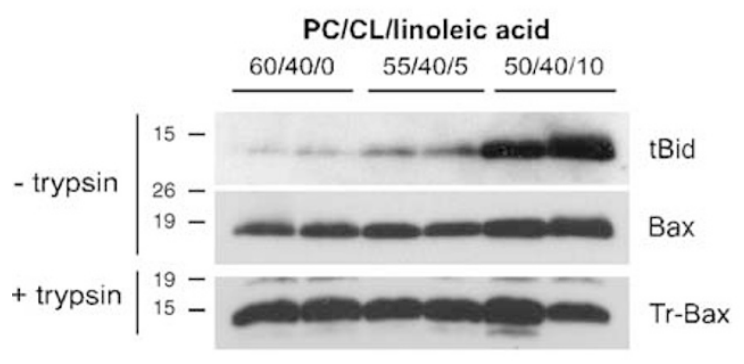

b

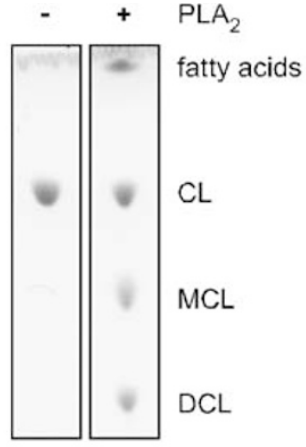

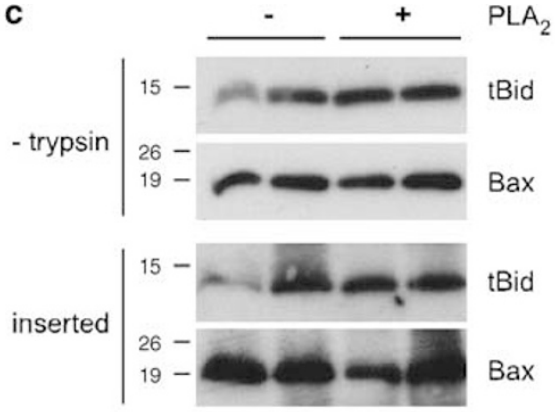

+ trypsin $\mid \begin{aligned} & 19- \\ & 15-\end{aligned}$
$\mathrm{PLA}_{2}$

Bid

Bax

Bid

Bax

$\operatorname{Tr}-\mathrm{Bax}$

Figure $5 \mathrm{CL}$ hydrolysis products do not prevent Bax insertion in vitro. (a) The effect of linoleic acid (0-10\%) on Bax activation in $\mathrm{PC} / \mathrm{CL}$ liposomes was examined in vitro. The binding of tBid and of Bax to liposomes (- trypsin) as well as the efficiency of Bax oligomerization ( + trypsin) were assessed by immunoblotting. (b, c) CL was incubated in the absence or presence of PLA . Lipids were analyzed by TLC (b) and used in combination with PC (PC/CL 60/40) to examine the efficiency of Bax activation in vitro (c). The binding and insertion of tBid and Bax were assessed, as well as the efficiency of Bax oligomerization. (a, c) Blots show duplicates and are representative of three independent experiments

of freezing and thawing cycles (F/T), or extruded liposomes through polycarbonate filters with pore diameters ranging from 800 to $200 \mathrm{~nm}$. Liposomes were reconstituted in a sucrose solution, iso-osmotic with the $\mathrm{KCl}$ buffer used in our assays, a procedure that did not affect the outcome of in vitro Bax activation assays (data not shown). Liposomes were visualized by microscopy after staining with $10-N$-nonylacridine orange (NAO, Figure 6a). F/T liposomes were heterogeneous in size, and large liposomes $(>5 \mu \mathrm{m}$ diameter) were often observed. With negative stain electron microscopy, a clear difference between the dimensions of the different populations of extruded liposomes was observed (Figure 6a). Dynamic light scattering, although less suitable for analyzing the size distribution of large particles, also showed that we had obtained distinct populations of liposomes, with mean hydrodynamic radii in agreement with the diameters of the pores of the filters (Supplementary Figure 3a).

When in vitro Bax activation assays were performed with these different preparations of liposomes, we observed that 
a

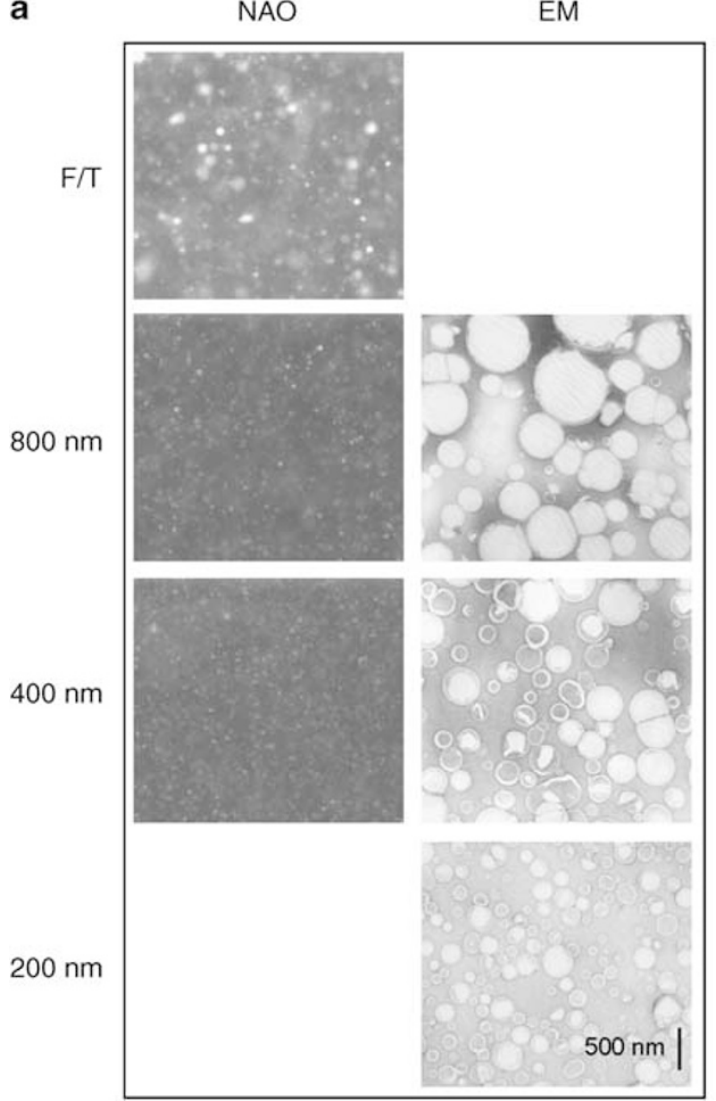

b
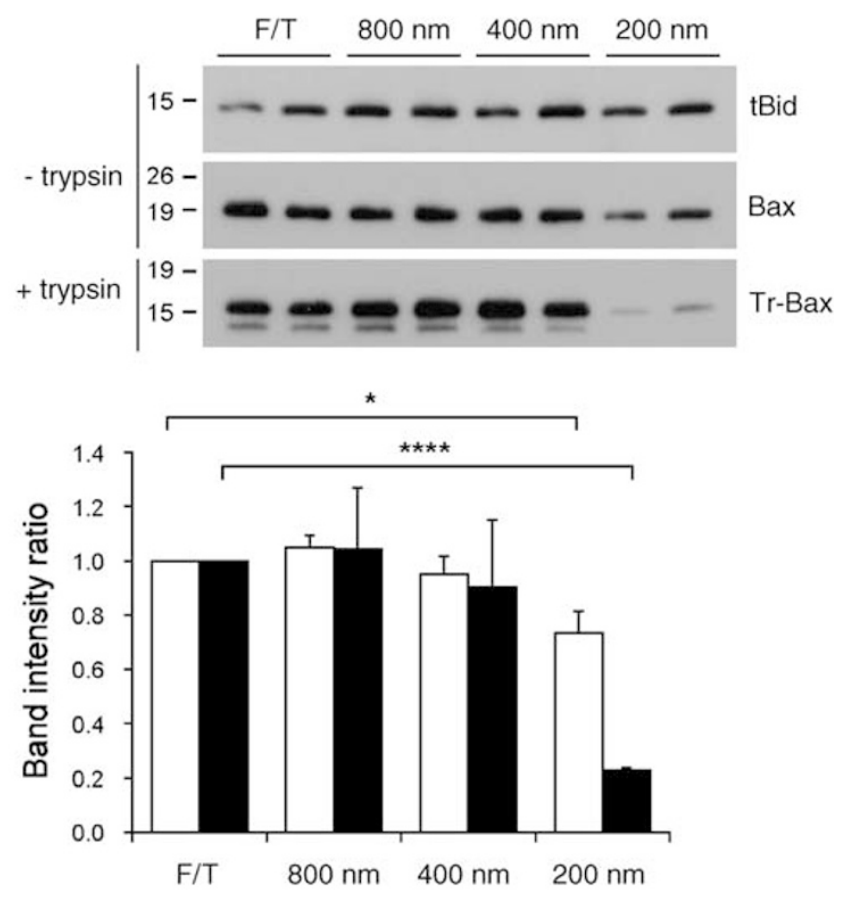

Figure 6 Small liposomes do not sustain Bax oligomerization in vitro. (a) MOM-like liposomes were prepared by F/T or were extruded through polycarbonate filters with defined pore sizes $(800,400$, and $200 \mathrm{~nm})$. Their size distribution was checked by staining with NAO and visualization under a microscope, or by negative stain electron microscopy (EM). (b) These liposomes were used in tBid-induced Bax activation assays in vitro to examine the efficiency of binding of tBid and Bax (- trypsin), and Bax oligomerization ( + trypsin). Blot shows duplicates. The intensities of the bands corresponding to Bax bound to the liposomes (white bars) and to Bax oligomers (black bars) were quantified by densitometric analysis, and ratios to the intensities obtained with $F / T$ liposomes were calculated. Values are means of four independent experiments \pm S.E.M. ${ }^{*} P<0.03,{ }^{* * * *} P<0.00001$

whereas Bax bound efficiently to large liposomes and oligomerized, with $200 \mathrm{~nm}$ liposomes, it bound slightly less efficiently and Tr-Bax was hardly detectable, indicating that Bax oligomerization was inhibited in small liposomes (Figure 6b). Similarly, Bax oligomerization was completely prevented in liposomes extruded through filters with $100 \mathrm{~nm}$ pores (data not shown). In contrast, tBid bound well to liposomes, independent of their size. All of these different liposomes pelleted efficiently, ruling out the possibility that the lack of Bax oligomerization resulted from the loss of small liposomes during the ultracentrifugation step (Supplementary Figure 3b). These experiments suggest that Bax does not preferentially oligomerize in mitochondrial fission sites because of the local curvature of the membrane.

\section{Discussion}

The importance of Bax and Bak for the so-called intrinsic pathway of apoptosis has been long known, since the observation that cells lacking Bax and Bak are resistant to many apoptotic insults. ${ }^{31}$ Under normal conditions, Bax exists as a soluble monomer, and Bak is already integrated in the MOM. Activation of the two proteins leads to their oligomerization in the lipid bilayer, resulting in MOM permeabilization. These processes need to be tightly controlled, as cytochrome $c$ release has often been shown to be the point of no return, leading to demise of the cell. ${ }^{32}$ Similarly to what has been reported for protein digestion, ${ }^{22,23}$ we show that artificially modifying the composition of mitochondrial membranes with $\mathrm{PLA}_{2}$ inhibits $\mathrm{Bax}$ and tBid insertion, underlining the role played by the lipid composition and/or the intrinsic properties of the MOM in these processes.

$\mathrm{CL}$ is a mitochondria-specific phospholipid that was repeatedly shown to play important roles in apoptosis. ${ }^{33}$ Many groups independently showed that $\mathrm{CL}$ or its metabolites are important for the activities of tBid and/or Bax, and to control the binding of cytochrome $c$ to the MIM. ${ }^{12,13,17,18,34}$ Rapidly following apoptosis induction, $\mathrm{CL}$ was shown to redistribute to the $\mathrm{MOM}^{35,36}$ and to be metabolized by peroxidation ${ }^{13}$ and hydrolysis, ${ }^{10}$ although the precise time course of events relative to Bax activation and MOM permeabilization is still unclear. Our results suggest that whereas a rise in the level of $\mathrm{CL}$ in the MOM would facilitate Bax activation, Bax oligomerization would be prevented by $\mathrm{CL}$ hydrolysis, suggesting that it occurs after Bax activation. 
Recently, decreasing CL levels in the MOM by expression of a dominant negative mutant of phospholipid scramblase 3 or with specific small hairpin RNAs (shRNAs) were shown to increase the resistance of cells to apoptosis-inducing agents. $^{35,37}$ Our in vitro experiments suggest that a PLA ${ }_{2}$ sensitive phospholipid present in low amounts in the MOM is essential for the early steps of Bax activation. CL appeared as the most likely candidate, since its low abundance in the MOM and its negative charge would be compatible with a high efficiency of hydrolysis by pancreatic $\mathrm{PLA}_{2}{ }^{24}$ Indeed, our in vitro studies show that $C L$ is required for Bax binding and oligomerization in isolated liposomes, independent of the recruitment of $\mathrm{tBid}$, and that Bax insertion is inhibited in $\mathrm{CL}$ deficient yeast mitochondria. Many doubts have, however, recently been raised about the importance of CL for Bax activation, essentially based on the observation that Bax activation can occur in the absence of CL. ${ }^{38-40}$ However, it is possible that under certain circumstances, other phospholipids, such as phosphatidic acid or some phosphoinositides, might replace $\mathrm{CL}$, and that what is important is not CL per se, but the properties it confers to the lipid bilayer. The human gene coding for CLS, the enzyme catalyzing the final step of CL biosynthesis, was recently identified, ${ }^{41,42}$ and in one study, the impact of downregulating the expression level of the enzyme using shRNAs on the sensitivity of cells to apoptosis was analyzed. ${ }^{42}$ Stable cell lines expressing these shRNAs and containing lower $\mathrm{CL}$ levels, as revealed by the amount of $\left[{ }^{3} \mathrm{H}\right]$ incorporated in $\mathrm{CL}$ after a $24 \mathrm{~h}$ labeling with $\left[{ }^{3} \mathrm{H}\right]$-palmitic acid, were selected. These clones were more susceptible to apoptosis than clones expressing control shRNAs. ${ }^{42}$ However, our results suggest that downregulating the expression of $C L S$ with shRNAs could impact on the rate of CL synthesis reflected in the incorporation efficiency of $\left[{ }^{3} \mathrm{H}\right]$-palmitic acid without altering total CL levels (Supplementary Figure 4). Accordingly, transient downregulation of CLS with shRNAs did not alter the efficiency of Bax activation both in vitro upon incubation of isolated mitochondria with tBid and following exposure of cells to actinomycin D or staurosporine (Supplementary Figure 5). Unfortunately, in their paper, Choi et al. did not measure total CL levels. ${ }^{42}$ Therefore, the significance of $\mathrm{CL}$ in mammalian cells for Bax activation and MOM permeabilization still needs to be further examined, even though the in vitro results on isolated mitochondria presented here further support the idea that CL, or the bilayer structure it creates, plays an important role in the process. Indeed, in comparison to other lipids, CL possesses the capacity to adopt both flat and hexagonal II phase structures, which could explain its enrichment in mitochondrial contact sites. The presence of CL might thus improve the flexibility of the bilayer required to accommodate insertion of Bax and formation of large Bax oligomers.

PE did not impact in vitro on Bax or tBid insertion, but at $20 \%$ drastically inhibited Bax oligomerization. Interestingly, PE was recently reported to inhibit the permeabilization of liposomes induced by oligomerized Bax. ${ }^{18}$ As PE did not impact on Bax insertion, the authors proposed that, because of its intrinsic negative curvature, PE directly inhibits opening of a lipidic pore. Our results, however, suggest that PE could also inhibit liposome permeabilization because of an inhibition of Bax oligomerization.
The sensitivity of the Bax activation process to characteristics of the lipid bilayer suggests that lipids constitute an additional mode of regulation of Bax activation. The early lipid modifications that occur following apoptosis induction ${ }^{10,13}$ or the interaction between $\mathrm{tBid}$ and the $\mathrm{MOM}^{11}$ could change the properties of the membrane in such a way that Bax oligomerization and MOM permeabilization would only then become possible. Lipids probably also contribute to Bax activation by favoring its insertion and oligomerization in certain regions of the MOM, and inhibiting them in others. The punctuate distribution of activated Bax in mitochondria and in particular its association with mitochondrial scission sites could be linked to interactions between Bax and mitochondrial fission proteins. ${ }^{19}$ Alternatively, and probably not exclusively, Bax could specifically interact with lipids concentrated in these constriction sites, such as CL whose structure might facilitate membrane fission events, or Bax localization could even result from its exclusion from ordered domains of the MOM.

Recently, an impressive number of Bax-interacting proteins have been identified, but the relevance of these interactions for controlling cell survival and death is questionable. ${ }^{3}$ Indeed, these proteins do not share a common domain or activity that could explain how they might control the conformational rearrangements of Bax. Moreover, when mapping experiments were undertaken, diverse regions of Bax were identified. Therefore, given the well-known stickiness of Bcl-2 family proteins, ${ }^{2}$ one might question whether controlling Bax activation is really the way they impact on cell survival and death. So far, the roles of lipids in regulating the conformational rearrangements of Bax and Bak have probably been underestimated. Bax and Bak have to remodel in a lipid environment, and many physical parameters defined by the precise composition of the bilayer (hydrophobic thickness, lateral pressure) might have an impact on the efficiency of their insertion and oligomerization. Therefore, understanding precisely how lipids contribute to the activation of Bax and Bak is certainly required to have a complete comprehension of these processes and to imagine new ways to impact on cell survival and death.

\section{Materials and Methods}

Cell culture and transfection. HeLa cells (European Collection for Cell Cultures) were grown in DME supplemented with $10 \% \mathrm{FCS}, 100 \mathrm{U} / \mathrm{ml}$ penicillin, $0.1 \mathrm{mg} / \mathrm{ml}$ streptomycin, $2 \mathrm{mM}$ glutamine, and maintained in $5 \% \mathrm{CO}_{2}$ at $37^{\circ} \mathrm{C}$. Culture plates were from Nunc; all other cell culture reagents were from Sigma.

Recombinant proteins. Recombinant His-tagged full-length Bax, caspase-8cleaved Bid (tBid) and His-Fis were purified as described previously., ${ }^{4,23,26}$

Mitochondria isolation. HeLa cells were scraped off from plates and washed in cold PBS. Mitochondria were purified following a previously described protocol, ${ }^{14}$ with slight modifications. In brief, cells were resuspended in MB buffer $(210 \mathrm{mM}$ mannitol, $70 \mathrm{~mm}$ sucrose, $1 \mathrm{~mm}$ EDTA, $10 \mathrm{~mm}$ Hepes, pH 7.4) containing protease inhibitors (Roche), and mechanically broken three times using a $2 \mathrm{ml}$ glass/glass Dounce homogenizer (Kontes) (20,15, and 15 strokes). Homogenates were cleared at $1500 \times g$ and mitochondria were spun down at $10000 \times g$. Protein concentrations were measured with a Bradford assay (Bio-Rad Laboratories) and processed for further tests.

PLA $_{2}$ hydrolysis. Isolated HeLa mitochondria $(1 \mu \mathrm{g} / \mu \mathrm{l})$ were incubated with $\mathrm{PLA}_{2}$ from porcine pancreas ( 0.3 or $1.5 \mu \mathrm{g} / \mathrm{ml}$, Sigma) in $\mathrm{KCl}$ buffer $(125 \mathrm{mM} \mathrm{KCl}$, $4 \mathrm{mM} \mathrm{MgCl}_{2}, 5 \mathrm{mM} \mathrm{KH}_{2} \mathrm{PO}_{4}, 10 \mathrm{mM}$ Hepes, $\mathrm{pH} 7.4$ ) in the presence of $0.5 \mathrm{mM}$ 
$\mathrm{CaCl}_{2}$, for $10 \mathrm{~min}$ at $30^{\circ} \mathrm{C}$. Reactions were stopped with $1 \mathrm{~mm} \mathrm{EGTA}$, and lipids were extracted for TLC analysis, or mitochondria were processed for further tests.

Pure CL $(2 \mu \mathrm{g} / \mu \mathrm{l})$ was hydrolyzed with $\mathrm{PLA}_{2}(3 \mu \mathrm{g} / \mathrm{ml})$ in $(50 / 50)$ water/EtOH, $5 \mathrm{~mm}$ tris pH $7.4,1 \mathrm{mM} \mathrm{CaCl}$, for $5 \mathrm{~min}$ at $30^{\circ} \mathrm{C}$. Reaction was stopped with EGTA $(2 \mathrm{mM})$; lipids were extracted, dried under nitrogen, and resuspended in $\mathrm{CHCl}_{3}$.

tBid-induced Bax insertion in isolated mitochondria. Mitochondria were resuspended at $1 \mu \mathrm{g} / \mu \mathrm{l}$ in $\mathrm{KCl}$ buffer supplemented with succinate $(5 \mathrm{mM})$ and EGTA $(0.5 \mathrm{~mm})$. tBid $(20$ or $100 \mathrm{nM})$ was added to the samples and a $12 \mathrm{~min}$ incubation was carried out at room temperature. Samples were centrifuged $(5 \mathrm{~min}$, $10000 \times g, 4^{\circ} \mathrm{C}$ ). Supernatants were recovered and analyzed by immunoblotting, and pellets were resuspended at $0.5 \mu \mathrm{g} / \mu \mathrm{l}$ in freshly made $\mathrm{Na}_{2} \mathrm{CO}_{3}(0.1 \mathrm{M})$; they were then incubated on ice for $30 \mathrm{~min}$ and spun for $30 \mathrm{~min}$ at $100000 \times \mathrm{g}, 4^{\circ} \mathrm{C}$. These pellets were analyzed by immunoblotting.

Lipid extraction and TLC analysis. Mitochondria (50 $\mu \mathrm{g}$ protein) or liposomes (50 $\mu \mathrm{g}$ lipid) were resuspended in $260 \mu \mathrm{l}$ of $0.9 \% \mathrm{NaCl}$ and $10 \mathrm{mM} \mathrm{HCl}$ aqueous solution. $\mathrm{MeOH}(666 \mu \mathrm{l})$ and $\mathrm{CHCl}_{3}(333 \mu \mathrm{l})$ were added, and samples were vortexed and incubated on ice for $10 \mathrm{~min} . \mathrm{CHCl}_{3}(333 \mu \mathrm{l})$ and $0.9 \% \mathrm{NaCl}$, $10 \mathrm{mM} \mathrm{HCl}(333 \mu \mathrm{l})$ were further added, and samples were again vortexed and incubated on ice for $10 \mathrm{~min}$. After a brief centrifugation $\left(5 \mathrm{~min}, 250 \times \mathrm{g}, 4^{\circ} \mathrm{C}\right)$, the organic phase was recovered and dried under nitrogen at $30^{\circ} \mathrm{C}$. Dried lipids were resuspended in a small volume of $\mathrm{CHCl}_{3}$ and spotted on silica gel $60 \mathrm{TLC}$ plates (Merck). Unless otherwise stated, plates were developed using a $\mathrm{CHCl}_{3} / \mathrm{MeOH} /$ water (43/17/2.7) mixture. Plates were air-dried, bathed in a copper (II) acetate solution (3\%) in $8 \% \mathrm{H}_{3} \mathrm{PO}_{4}$, and heated to $120^{\circ} \mathrm{C}$ for 5-15 min. Commercial lipids were used to identify the spots. Their intensity was quantified using ImageJ (http:// rsb.info.nih.gov/ij/).

Immunoblotting and immunocytochemistry. Samples were separated in polyacrylamide gels and transferred to Hybond-P membranes (Amersham Pharmacia). Immunoblotting was performed with the following antibodies: Bax (BD-Pharmingen), tBid (R\&D Systems), Fis and caspase-3 (Alexis Biochemicals), polyclonal cytochrome $c$, Smac/DIABLO (ProSci Inc.), Bcl- $\mathrm{X}_{\mathrm{L}}$ (Transduction Laboratories), polyclonal SLP-2 (da Cruz, in preparation), Bak (Upstate Biotechnology), actin (Sigma), aldolase (Chemicon), and yeast Hsp-82 (gift from $D$ Picard). ${ }^{43}$ Secondary antibodies (anti-mouse and anti-rabbit from DakoCytomation, anti-goat from Santa Cruz Biotechnology, and anti-chicken) were coupled to horseradish peroxidase and signals were detected by ECL.

Densitometric analysis of the bands was performed by analyzing the films using ImageJ (http://rsb.info.nih.gov/ij). Statistical significance was assessed using a paired Student's $t$-test.

Preparation of liposomes and in vitro Bax oligomerization assay. Egg yolk PC, bovine heart $\mathrm{CL}$, bovine brain $\mathrm{PE}$, bovine liver $\mathrm{PI}, \mathrm{CHO}$ and linoleic acid were from Sigma. Lipids were mixed (w/w ratios) and dried under nitrogen at $30^{\circ} \mathrm{C}$. They were incubated with $\mathrm{KCl}$ buffer for $20 \mathrm{~min}$ on a rotating wheel, and completely resuspended by gentle vortexing. Five cycles of freezing in liquid nitrogen and thawing at $30^{\circ} \mathrm{C}$ were performed. Bax activation assays were then undertaken. Unless otherwise stated, liposomes $(1 \mu \mathrm{g} / \mu \mathrm{l}$ in $\mathrm{KCl}$ buffer) were incubated with recombinant His-Bax $(100 \mathrm{~nm})$ and tBid $(10 \mathrm{~nm})$ for $30 \mathrm{~min}$ at $30^{\circ} \mathrm{C}$. Unbound proteins were removed by ultracentrifugation $\left(30 \mathrm{~min}, 180000 \times \mathrm{g}, 10^{\circ} \mathrm{C}\right)$. The pellets were then resuspended in $\mathrm{KCl}$ buffer; half of each pellet was kept to analyze the binding of Bax and tBid, while the other half was incubated with trypsin $(0.17 \mathrm{mg} / \mathrm{ml})$ for $2 \mathrm{~h}$ at $30^{\circ} \mathrm{C}$. Reactions were stopped with trypsin inhibitor $(1.4$ $\mathrm{mg} / \mathrm{ml}$, Sigma). The amounts of proteins bound to liposomes and the fraction of Bax resistant to trypsin digestion were analyzed by immunoblotting. To test the insertion of $\mathrm{Bax}$ or tBid in the lipid bilayers, the pellets were instead incubated with $\mathrm{Na}_{2} \mathrm{CO}_{3}$ $(0.1 \mathrm{M})$ for $30 \mathrm{~min}$ on ice. After ultracentrifugation, the pellets were analyzed by immunoblotting.

Isolation of yeast mitochondria. The homozygous diploid deletion strain $\Delta c r d 1$ and the isogenic wild-type strain BY4743 (Euroscarf, Germany) were grown in YPD medium following classical procedures. In logarithmic phase, spheroplasts were prepared as described ${ }^{44}$ and mitochondria were purified essentially according to Iverson et al. ${ }^{27}$ They were then incubated at $1 \mu \mathrm{g} / \mu \mathrm{l}$ in $\mathrm{KCl}$ buffer supplemented with succinate $(5 \mathrm{mM})$ and EGTA $(0.5 \mathrm{mM})$ with recombinant His-Bax $(100 \mathrm{nM})$ in the absence or presence of tBid $(20 \mathrm{nM})$ at room temperature. After centrifugation at $10000 \times g$ to remove any unbound proteins, the pellets $(0.5 \mu \mathrm{g} / \mu \mathrm{l})$ were incubated for $30 \mathrm{~min}$ on ice in $\mathrm{Na}_{2} \mathrm{CO}_{3}(0.1 \mathrm{M})$. After a $30 \mathrm{~min}$ ultracentrifugation at $100000 \times g$, Bax and tBid insertion in the membrane pellets were examined by immunoblotting.

Extrusion of liposomes. As described above, liposomes of defined compositions were prepared, but in a sucrose solution, iso-osmotic with the $\mathrm{KCl}$ buffer. After five $F / T$ cycles, liposomes were extruded 10 times through polycarbonate filters with pores of $800,400,200$, or $100 \mathrm{~nm}$ diameter (Reichelt Chemietechnik, Heidelberg, Germany). Equal amounts of lipids were used in in vitro Bax activation assays, following the protocol described above. After the ultracentrifugation step, $10 \%$ of the pellets were kept for lipid extraction and TLC analysis.

Visualization of liposomes. NAO $(5 \mu \mathrm{M})$ was added to liposomes, which were then visualized using a Zeiss Axiophot microscope. Pictures were taken using a Qlmaging Retiga Ex camera with Openlab 3.5.0 (Improvision).

Carbonated formvar-coated copper electron microscopy grids were glow discharged and covered with $10 \mu \mathrm{l}$ drop of liposome solution for $45 \mathrm{~s}$, then dried by placing their side on a filter paper. The grids were then rapidly rinsed with two drops of water, and successively placed on two $200 \mu$ ldrops of uranyl acetate $2 \%$, for 1 and $30 \mathrm{~s}$. The excess liquid was then removed on a filter paper. The grids were examined at $60 \mathrm{kV}$ using a Philips M400 transmission electron microscope.

Dynamic light scattering. Extruded liposomes were diluted in $\mathrm{KCl}$ buffer to $0.25 \mu \mathrm{g} / \mu \mathrm{l}$ and analyzed by DLS at $90^{\circ}$ with ALV-CGS3 (Langen, Germany). This compact goniometer system uses a $20 \mathrm{~mW}$ He-Ne laser of wavelength $633 \mathrm{~nm}$.

Acknowledgements. We thank Professor Michal Borkovec, Dr. Paolo Galletto, and Michal Skarba for help and guidance with dynamic light scattering. We are grateful to Professor Valerian Kagan and Dr. Yulia Tyurina for providing us with some MCL and DCL. We also thank Dr. Christoph Bauer and the NCCR Bioimaging Platform for help with negative stain electron microscopy. We are thankful to Dr. Yves Mattenberger for his advice and guidance with the quantitative PCR experiments, and Professor Didier Picard for providing us with anti-Hsp82 antibodies. We would finally like to express our gratitude to Professor Richard Epand for the helpful discussions, and Professor EAC Lucken and Dr. Julien Häsler for their comments and interesting discussions about this paper. This work was funded by the Swiss National Science Foundation (subsidy 3100A0-109419/1), OncoSuisse Trust, and the Geneva Department of Education.

1. Chipuk JE, Bouchier-Hayes L, Green DR. Mitochondrial outer membrane permeabilization during apoptosis: the innocent bystander scenario. Cell Death Differ 2006; 13: 1396-1402.

2. Borner $\mathrm{C}$. The Bcl-2 protein family: sensors and checkpoints for life-or-death decisions. Mol Immunol 2003; 39: 615-647.

3. Lucken-Ardjomande $S$, Martinou JC. Regulation of $\mathrm{Bcl}-2$ proteins and of the permeability of the outer mitochondrial membrane. C R Biol 2005; 328: 616-631.

4. Desagher S, Osen-Sand A, Nichols A, Eskes R, Montessuit S, Lauper S et al. Bid-induced conformational change of Bax is responsible for mitochondrial cytochrome $c$ release during apoptosis. J Cell Biol 1999; 144: 891-901.

5. Goping IS, Gross A, Lavoie JN, Nguyen M, Jemmerson R, Roth $\mathrm{K}$ et al. Regulated targeting of BAX to mitochondria. J Cell Biol 1998; 143: 207-215.

6. Hsu YT, Wolter KG, Youle RJ. Cytosol-to-membrane redistribution of Bax and Bcl-X(L) during apoptosis. Proc Natl Acad Sci USA 1997; 94: 3668-3672.

7. Makin GW, Corfe BM, Griffiths GJ, Thistlethwaite A, Hickman JA, Dive C. Damage-induced Bax N-terminal change, translocation to mitochondria and formation of Bax dimers/ complexes occur regardless of cell fate. EMBO J 2001; 20: 6306-6315.

8. Upton JP, Valentijn AJ, Zhang L, Gilmore AP. The N-terminal conformation of Bax regulates cell commitment to apoptosis. Cell Death Differ 2007; 14: 932-942.

9. Leber B, Lin J, Andrews DW. Embedded together: the life and death consequences of interaction of the Bcl-2 family with membranes. Apoptosis 2007; 12: 897-911.

10. Esposti MD, Cristea IM, Gaskell SJ, Nakao Y, Dive C. Proapoptotic Bid binds to monolysocardiolipin, a new molecular connection between mitochondrial membranes and cell death. Cell Death Differ 2003; 10: 1300-1309.

11. Kim TH, Zhao Y, Ding WX, Shin JN, He X, Seo YW et al. Bid-cardiolipin interaction mitochondrial contact site contributes to mitochondrial cristae reorganization and cytochrome $c$ release. Mol Biol Cell 2004; 15: 3061-3072.

12. Lutter M, Fang M, Luo X, Nishijima M, Xie X, Wang X. Cardiolipin provides specificity fo targeting of tBid to mitochondria. Nat Cell Biol 2000; 2: 754-761. 
13. Kagan VE, Tyurin VA, Jiang J, Tyurina YY, Ritov VB, Amoscato AA et al. Cytochrome $c$ acts as a cardiolipin oxygenase required for release of proapoptotic factors. Nat Chem Biol 2005; 1: 223-232

14. Parone PA, James DI, Da Cruz S, Mattenberger Y, Donze O, Barja F et al. Inhibiting the mitochondrial fission machinery does not prevent Bax/Bak-dependent apoptosis. Mol Cell Biol 2006; 26: 7397-7408.

15. Scorrano L, Ashiya M, Buttle K, Weiler S, Oakes SA, Mannella CA et al. A distinct pathway remodels mitochondrial cristae and mobilizes cytochrome $c$ during apoptosis. Dev Cell 2002; 2: 55-67.

16. Basanez G, Sharpe JC, Galanis J, Brandt TB, Hardwick JM, Zimmerberg J. Bax-type apoptotic proteins porate pure lipid bilayers through a mechanism sensitive to intrinsic monolayer curvature. J Biol Chem 2002; 277: 49360-49365.

17. Kuwana T, Mackey MR, Perkins G, Ellisman MH, Latterich M, Schneiter R et al. Bid, Bax and lipids cooperate to form supramolecular openings in the outer mitochondrial membrane. Cell 2002; 111: 331-342.

18. Terrones $\mathrm{O}$, Antonsson B, Yamaguchi H, Wang HG, Liu Y, Lee RM et al. Lipidic pore formation by the concerted action of pro-apoptotic BAX and tBID. J Biol Chem 2004; 279 30081-30091.

19. Karbowski M, Lee YJ, Gaume B, Jeong SY, Frank S, Nechushtan A et al. Spatial and temporal association of Bax with mitochondrial fission sites, Drp1, and Mfn2 during apoptosis. J Cell Biol 2002; 159: 931-938.

20. Choi SY, Huang P, Jenkins GM, Chan DC, Schiller J, Frohman MA. A common lipid links Mfn-mediated mitochondrial fusion and SNARE-regulated exocytosis. Nat Cell Biol 2006; 8: $1255-1262$.

21. Roux A, Cuvelier D, Nassoy P, Prost J, Bassereau P, Goud B. Role of curvature and phase transition in lipid sorting and fission of membrane tubules. EMBO J 2005; 24: 1537-1545.

22. Bellot G, Cartron PF, Er E, Oliver L, Juin P, Armstrong LC et al. TOM22, a core component of the mitochondria outer membrane protein translocation pore, is a mitochondrial receptor for the proapoptotic protein Bax. Cell Death Differ 2006; 14: 785-794.

23. Roucou X, Montessuit S, Antonsson B, Martinou JC. Bax oligomerization in mitochondria membranes requires tBid (caspase-8-cleaved Bid) and a mitochondrial protein. Biochem $\mathrm{J}$ 2002; 368: 915-921.

24. Hovius R, Thijssen J, van der Linden P, Nicolay K, de Kruijff B. Phospholipid asymmetry of the outer membrane of rat liver mitochondria. Evidence for the presence of cardiolipin on the outside of the outer membrane. FEBS Lett 1993; 330: 71-76.

25. Tyurin VA, Tyurina YY, Osipov AN, Belikova NA, Basova LV, Kapralov AA et a. Interactions of cardiolipin and lyso-cardiolipins with cytochrome $c$ and tBid: conflict or assistance in apoptosis. Cell Death Differ 2007; 14: 872-875.

26. Lucken-Ardjomande S, Montessuit S, Martinou JC. Bax activation and stress-induced apoptosis delayed by the accumulation of cholesterol in mitochondrial membranes. Cell Death Differ 2007, doi:10.1038/sj.cdd.4402280.

27. Iverson SL, Enoksson M, Gogvadze V, Ott M, Orrenius S. Cardiolipin is not required for Bax-mediated cytochrome $c$ release from yeast mitochondria. J Biol Chem 2004; 279 . $1100-1107$.
28. Antonsson B, Montessuit S, Lauper S, Eskes R, Martinou JC. Bax oligomerization is required for channel-forming activity in liposomes and to trigger cytochrome $c$ release from mitochondria. Biochem J 2000; 345 (Part 2): 271-278.

29. Ingerman E, Perkins EM, Marino M, Mears JA, McCaffery JM, Hinshaw JE et al. Dnm1 forms spirals that are structurally tailored to fit mitochondria. J Cell Biol 2005; 170 : 1021-1027.

30. Daum G. Lipids of mitochondria. Biochim Biophys Acta 1985; 822: 1-42.

31. Wei MC, Zong WX, Cheng EH, Lindsten T, Panoutsakopoulou V, Ross AJ et al. Proapoptotic $\mathrm{BAX}$ and $\mathrm{BAK}$ : a requisite gateway to mitochondrial dysfunction and death. Science 2001; 292: 727-730

32. Kroemer G, Martin SJ. Caspase-independent cell death. Nat Med 2005; 11: 725-730.

33. Gonzalvez F, Gottlieb E. Cardiolipin: setting the beat of apoptosis. Apoptosis 2007; 12 : 877-885.

34. Ott M, Robertson JD, Gogvadze V, Zhivotovsky B, Orrenius S. Cytochrome c release from mitochondria proceeds by a two-step process. Proc Natl Acad Sci USA 2002; 99: 1259-1263.

35. He Y, Liu J, Grossman D, Durrant D, Sweatman T, Lothstein L et al. Phosphorylation of mitochondrial phospholipid scramblase 3 by protein kinase $\mathrm{C}$-delta induces its activation and facilitates mitochondrial targeting of tBid. J Cell Biochem 2007; 101: 1210-1221.

36. Qi L, Danielson ND, Dai Q, Lee RM. Capillary electrophoresis of cardiolipin with on-line dye interaction and spectrophotometric detection. Electrophoresis 2003; 24: 1680-1686.

37. Van Q, Liu J, Lu B, Feingold KR, Shi Y, Lee RM et al. Phospholipid scramblase-3 regulates cardiolipin de novo biosynthesis and its resynthesis in growing HeLa cells. Biochem J2007; 401: 103-109.

38. Hehl $A B$, Regos A, Schraner E, Schneider A. Bax function in the absence of mitochondria in the primitive protozoan Giardia lamblia. PLOS ONE 2007; 2: e488.

39. Scorrano L, Oakes SA, Opferman JT, Cheng EH, Sorcinelli MD, Pozzan T et al. BAX and $B A K$ regulation of endoplasmic reticulum $\mathrm{Ca}^{2+}$ : a control point for apoptosis. Science 2003; 300: $135-139$

40. Werneburg NW, Guicciardi ME, Bronk SF, Kaufmann SH, Gores GJ. Tumor necrosis factor-related apoptosis-inducing ligand activates a lysosomal pathway of apoptosis that is regulated by Bcl-2 proteins. J Biol Chem 2007; 282: 28960-28970.

41. Chen $D$, Zhang $X Y$, Shi $Y$. Identification and functional characterization of hCLS1, a human cardiolipin synthase localized in mitochondria. Biochem J 2006; 398: 169-176.

42. Choi SY, Gonzalvez F, Jenkins GM, Slomianny C, Chretien D, Arnoult D et al. Cardiolipin deficiency releases cytochrome $c$ from the inner mitochondrial membrane and accelerates stimuli-elicited apoptosis. Cell Death Differ 2007; 14: 597-606.

43. Louvion JF, Warth R, Picard D. Two eukaryote-specific regions of Hsp82 are dispensable for its viability and signal transduction functions in yeast. Proc Natl Acad Sci USA 1996; 93: 13937-13942.

44. Daum G, Bohni PC, Schatz G. Import of proteins into mitochondria. Cytochrome $b_{2}$ and cytochrome $c$ peroxidase are located in the intermembrane space of yeast mitochondria. J Biol Chem 1982; 257: 13028-13033.

Supplementary Information accompanies the paper on Cell Death and Differentiation website (http://www.nature.com/cdd) 